\title{
IROS 2018 Fan Challenge - Team DLR Augsburg
}

\author{
Manfred Schönheits ${ }^{1}$
}

\author{
Alfons Schuster ${ }^{1}$
}

\author{
Philipp Gänswürger ${ }^{1}$
}

Lars Larsen $^{1}$

\begin{abstract}
It's a hot summer in 2021 and the blistering sun is shining upon Madrid. You are enjoying some tinto de verano on your terraza. Sizzling in the scorching heat, you are trying to relax. With a simple gesture you call your robotic assistant to help you cool down a little bit. Without further ado, your robot provides some relaxing shade holding a parasol for you, picks up a fan autonomously and starts waving it and the gentle breeze brings you some light relief.
\end{abstract}

\section{INTRODUCTION}

At the German Aerospace Center (DLR) in Augsburg we are an interdisciplinary team of computer scientists, engineers and physicists. We work at the center for LightweightProduction-Technology (ZLP) which is a facility for applied research about process technology and automation for lightweight materials and components with a strong focus on the aerospace industry. We manufacture demonstrators, such as aircraft fuselages, boosters for the Ariane rocket or helicopter structures, with a Technology Readiness Level (TRL) up to six. Because of the size of the parts, the weight of industrial end-effectors and the variety of processes we make use of industrial robots heavily. The biggest facility is called multifunctional cell [6] (see fig. 1). It is a gantry robotic system which is $30 \mathrm{~m}$ long, $15 \mathrm{~m}$ wide and $7 \mathrm{~m}$ high. The workspace is shared by seven ceiling mounted robots. In all our projects the industrialization of the process is always an important aspect. Due to our background we have chosen a very pragmatic approach for the implementation of the IROS 2018 fan challenge. It was important for us to develop a very stable process which we can use many standard parts for.

Section II is about the early stage of our development and the rapid prototyping we did that laid the foundation for our system. An overview of our concept and the system architecture follows in section III. The most important design aspects of the end-effector we built are then depicted in section IV. In section V we describe how we implemented our camera-based fan detection component. This is followed by an explanation of the overall process in section VI.

\section{EARLY CONSIDERATIONS AND PROTOYPING}

First, in a concept phase, we examined how the fan can be opened manually by a human. We have found out that the fan requires a highly complex dexterity that we consider to be not trivial to implement with state-of-the-art robotic

\footnotetext{
${ }^{1}$ Manfred Schönheits, Alfons Schuster, Philipp Gänswürger and Lars Larsen are at the German Aerospace Center (DLR), Center for Lightweight-Production-Technology (ZLP), Am Technologiezentrum 4, D-86159 Augsburg, Germany \{manfred.schoenheits, alfons.schuster, philipp.gaenswuerger,

lars.larsen\} ddlr.de
}

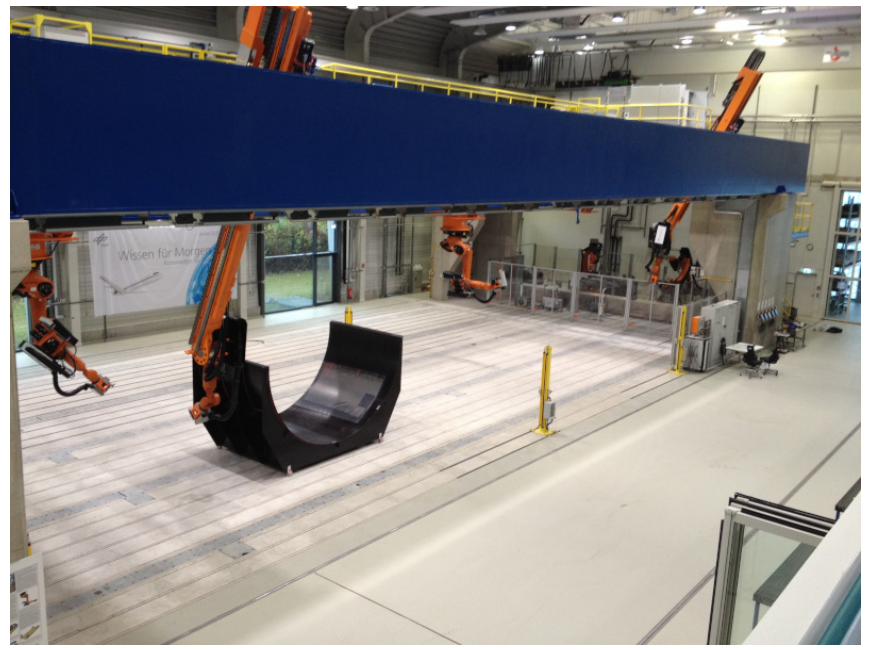

Fig. 1. Multifunctional robotic cell at the DLR Augsburg with ceiling mounted robots on linear tracks. On the shop floor a tooling of the lower fuselage of an Airbus A320 with two meter length has been positioned.

hands. It turned out that a safe opening and closing of the fan is not even easy for an untrained person by hand - the fan can easily be loosen or dropped and is hard to be opened completely. That is why we decided to develop a design that is based on the kinematics of the fan instead of mimicking the manual human process. After a brief brainstorming very quickly the idea was born to grasp the fan at the side surfaces with vacuum cups. In a first step suitable vacuum cups were selected by testing different products. After some tests a model was chosen which is in diameter slightly smaller than the side surface of the fan. The vacuum cups are made of silicone material and have a lip that adapts very well to the shape of the fan and thus enable very stable gripping. Figure 2 shows the prototype of the gripper. It consists of a bar with two boreholes where two blue pressure hoses are put through on which the blue vacuum cups are fixated. One can see that the vacuum cups can grip the fan laminar in the front and in the back near the rotation center where the lips of the cups clasp around to guarantee the vacuum.

After the initial test of the gripping principle a gripper with integrated kinematic has been build of LEGO stones. Figure 3 shows the gripper with two jaws which hold the fan from both sides. After the fan is fixated (see left figure) the rear jaw rotates about the dorn of the fan to open it. The LEGO gripper is equipped with the same vacuum cups which were identified before. After the successful test of the gripper prototype the next step was to develop and build an end-effector for the robot. 

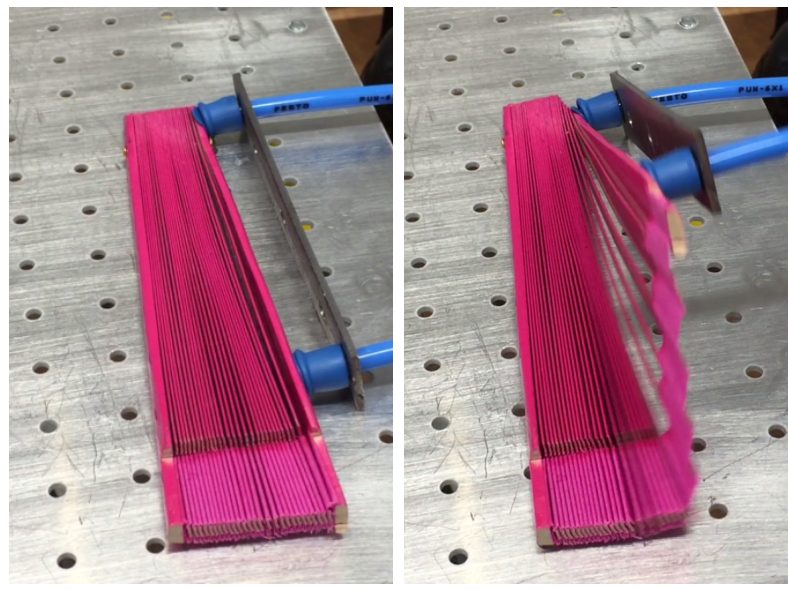

Fig. 2. Initial prototype of gripper jaw.
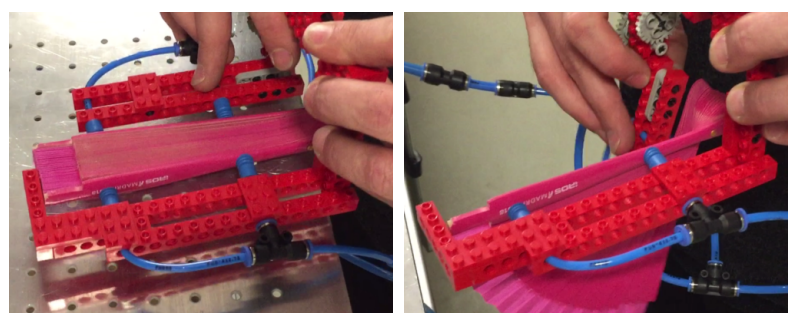

Fig. 3. Second prototype of gripper with kinematic.

\section{CONCEPT AND SYSTEM ARCHITECTURE}

One of the aspects of the challenge was to grasp the fan from a table autonomously. That raises the question for a suitable localization method. We decided to use a camerabased vision system because due to the field of view of the camera, the localization should be possible with only a single one-shot measurement which we considered to be faster than other methods that involve searching or scanning the area (e.g. tactile sensing or 2D laser profile scanners). Moreover, we already have significant experience and expertise with camera-based vision systems. We mounted the camera in a fixed position above the table to simplify the calibration because this avoids hand-eye-calibration.

The manipulator we used is a KUKA LBR iiwa 14 R820 [7]. A Robotiq 2-finger 140 adpative robot gripper [10] served as a basis for our end-effector. We used those devices mainly because they were available, but we think basically the whole system could also be built with different kinematics and grippers (e.g. a 4-DOF palletizing robot and a simple parallel gripper could suffice).

In order to automate a kinematic like the prototype introduced in section II, we had to solve the following challenge: The axis of rotation of the actuator has to be aligned with the axis of rotation of the fan. When the fan is put on the table, the axis is only a few millimeters above the surface. We came up with a solution to overcome this problem by using two split-gearwheels that give enough clearance towards the ground and also provide means to adjust the speed and degree

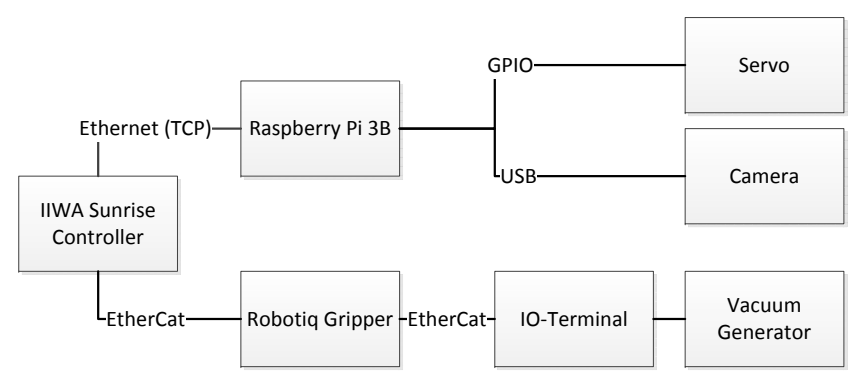

Fig. 4. System Architecture

of opening by altering the gear ratio. We decided to 3Dprint the gearwheels because we needed a custom non-stock design and wanted to do fast iterations. Figure 4 shows a schematic of the system. Our vision system runs on a Raspberry Pi 3 and communicates with the robot over TCP. The Servo is also driven by the Raspberry and is controlled by a separate process that also talks to the robot over TCP. The Robotiq gripper and the vacuum generator are interfaced through an Ethercat-Bus [3].

\section{MECHANICAL DESIGN}

The basic idea for the design of the gripper for the fan was to keep it as simple as possible and nevertheless very reliable while satisfying the kinematics of the fan. The Robotiq twofinger gripper could be directly mounted to the iiwa and adapted for the fan handling process. For the grasping of the fan the mechanism of the gripper was beneficial, as its two fingers describe a slight curve from open to close position. That made it possible to place the end-effector shortly above the surface in gripping position, which avoids having to touch the ground - which is an undesirable thing in spite of the LBR iiwa's programmable sensitivity. That is why we did not incorporate physical contact into the design of the endeffector in terms of e.g. stability and flexibility.

By measuring the opening angle of the fan, we decided to remain slightly below the measured 172 degrees to prevent overtwisting. The movement itself is actuated by a RC car servo with 120 degrees angle of rotation, so a gear transmission ratio of $1.4167: 1$ has been chosen.

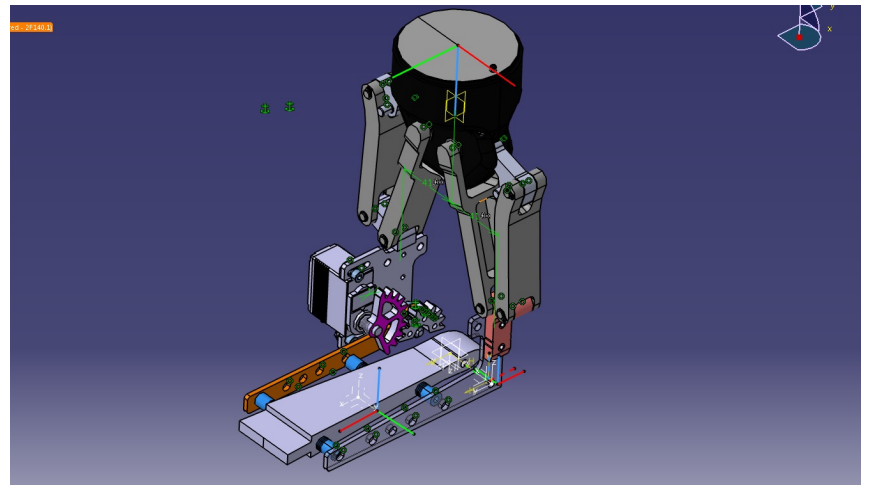

Fig. 5. Final end-effector design. 

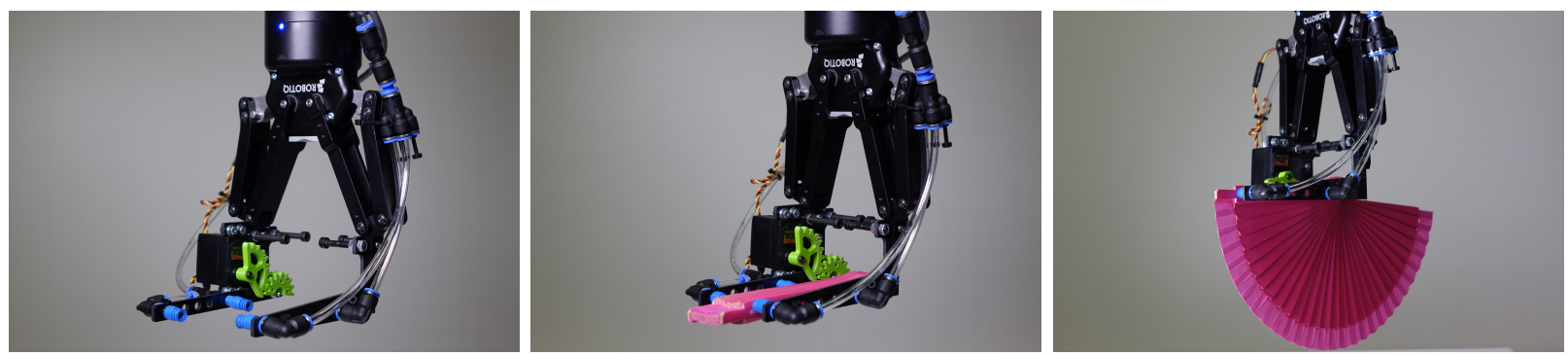

Fig. 6. Photos of the developed end-effector at different stages.

The momentum is transmitted with 3D printed gear wheels that are directly attached to the servo and the moving jaw. For a smooth movement, the minimum number of teeth of the smaller gear wheel was set to 10 . Multiplying the 1.4167 ratio by 10 , we got 14 teeth for the bigger gear wheel. A simple steel sleeve was manufactured on the turning machine for the pivot point and glued to one of the water jet cutted carbon plates that carry the suction cups and the servo. Overall, there were five parts to produce for the final endeffector design shown in fig. 5. With two adjustable screws, the close position of the two fingers is limited to the width of the fan. The screws can be seen in fig. 6 directly above the servo. Although the Robotiq gripper offers position control and basic contact detection, we decided to do this to prevent the fan from being damaged by squeezing (e.g. through a programming error) under all circumstances as we only had one sample available. Knowing the coordinates of the fan, the pivot of the moving jaw of the end-effector can be aligned with the pivot of the fan by adding a constant offset.

\section{FAN DETECTION}

In order to enable autonomous gripping of the fan, we implemented a fan detection system based upon a lowcost but flexible smart-cam system. An IDS $\mu$-eye 5MP color USB camera [5] was connected to a Raspberry Pi model $3 \mathrm{~b}$. To enable flexible image processing the latest version of Willow-Garage's OpenCV [9] was compiled for supporting the Pi's eight cores. Since the $\mu$-eye was not working properly with OpenCV's built in capture capabilities the freely available pymuеye python-wrapper was used to integrate image acquisition. The Pi also runs a TCP-server and detection is triggered on reception of a TCP command string issued by the robot.

\section{A. Detection}

Our approach for detection was converting to grayscale, thresholding, border detection by Canny's algorithm [2] and contour detection by Suzuki [11] as shown in fig. 7 using the standard OpenCV-methods.

It was found that the camera's full 5MP resolution is not necessary for the given problem and leads to performance problems as well as to interrupted contours, what leads to complications when trying to eliminate false positive detections. It turned out that acquiring VGA (640x480) images that are scaled down to $400 \times 300$ pixels yields most stable results at sufficient accuracy and good speed. In order to maximize contrast when converting to grayscale we examined the histograms channelwise with the clear yet not unexpected result to use the green channel for the pink fan for best contrast (fig. 8).

Having done this, we picked only the contours with an area of approximately the fan's pixel footprint thus eliminating false positives. Then we applied a Hough [1] line finder algorithm in order to detect the fan's long side borders (fig. 9). From the contour's center of gravity we drop the perpendicular on the Hough-lines and finally identify the correct border by intersecting both borders and taking the one with the bigger absolute angle between base point and intersection.

\section{B. Calibration}

Once the detection is complete, the 2D-image coordinates of the reference point on the fan's desired side facing the gripper as well as the fan's orientation have to be transformed into useful 3D robot coordinates. Since the problem is only 2D we decided not to perform hand-eye calibration but instead used a perspective transform [4] to transform two 2D image points into the robot's coordinate system yielding grip point and grip angle assuming that the table is in the $x-y$ plane with $\mathrm{z}=0$. Calibration is done straight forward by taking an image with four reference points $\left(x_{1}, y_{1}\right) . .\left(x_{4}, y_{4}\right)$, capturing the four points $\left(\tilde{x_{1}}, \tilde{y_{1}}\right) . .\left(\tilde{x_{4}}, \tilde{y_{4}}\right)$ with the robot using a measuring tip and afterwards obtaining the transformation by calling OpenCV's findHomography method. The transformed result in the robot's coordinate system is subsequently passed to the robot controller for the further gripping by a TCP string in reply of the robot's request.

\section{PROCESS CONTROL}

Due to the nature of the setup, the overall process is comparably simple. The process logic runs on the robot controller and is implemented as a Java application on the Sunrise platform [8]. Figure 10 shows a schematic of the process. At the beginning, the robot is in a starting position. Through sending a TCP packet to the fan detection on the Raspberry, the system obtains the coordinates of the fan. The application then uses the coordinates to plan a path for the robot. The path planning algorithm we implemented is rather simple and just calculates a single spline curve. The robot's current position is the starting point of the curve, 


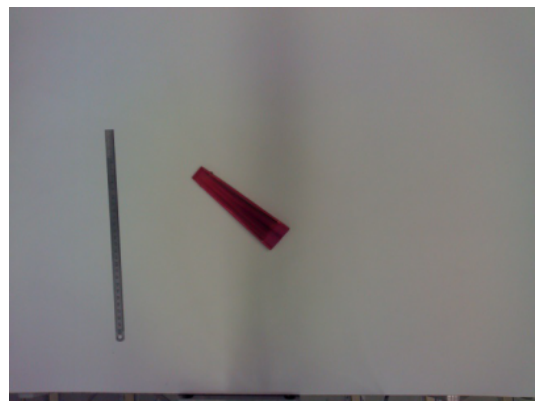

(a)

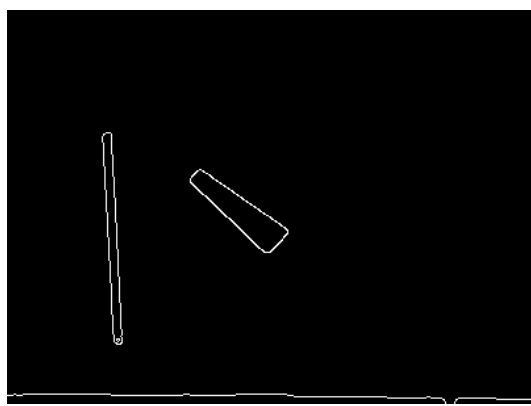

(d)

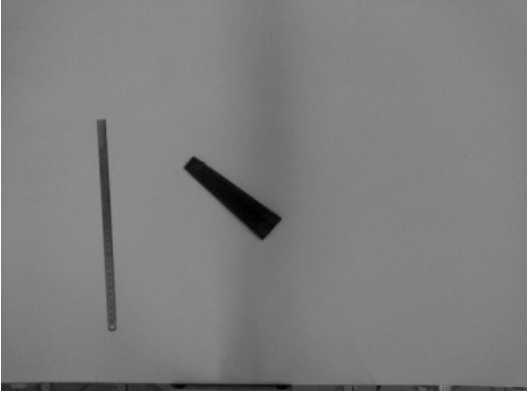

(b)

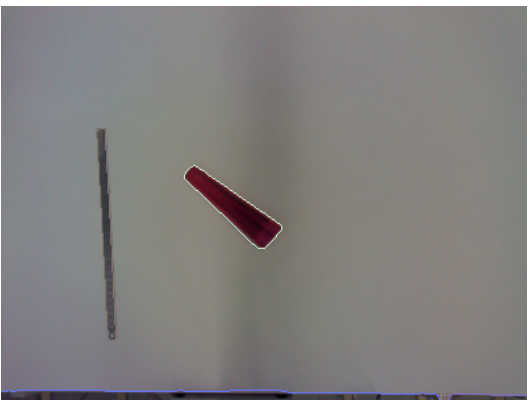

(e)

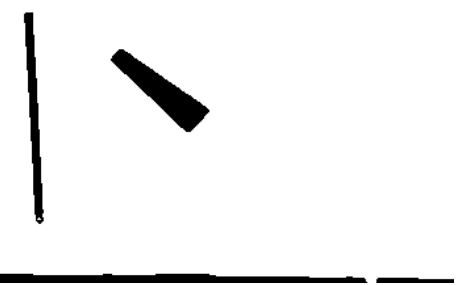

(c)

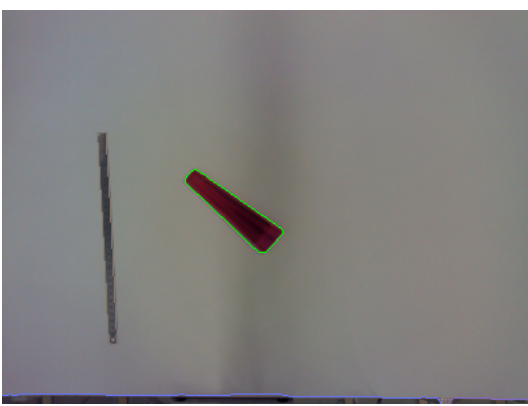

(f)

Fig. 7. Detection steps: (a) Original Image, (b) Green Channel, (c) Thresholded image, (d) Contour detection by Canny's algorithm, (e) Contours, (f): Proper size contour (green)

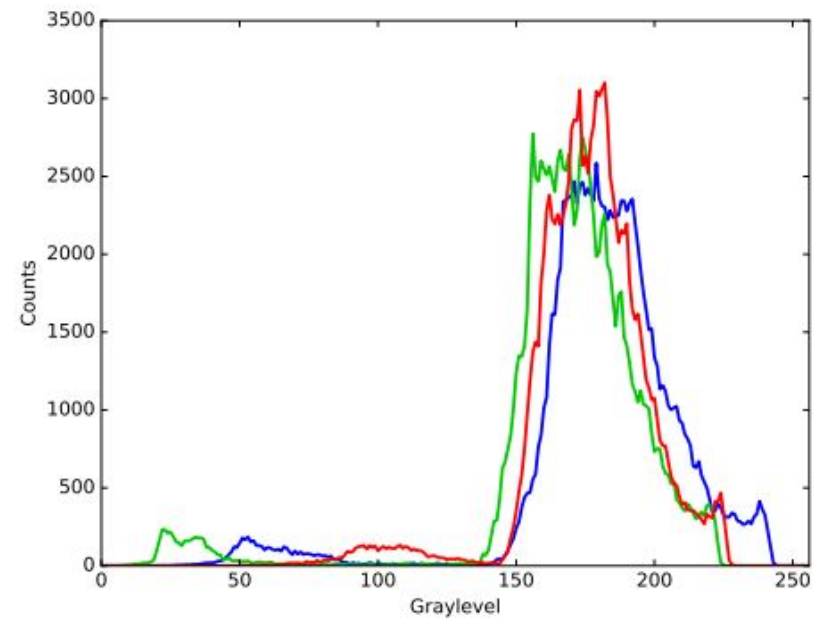

Fig. 8. Channelwise image histogram

the fan coordinates are used to determine the target position. Two intermediate auxiliary points are calculated to shape the curve. When the robot reached the target position, the gripper closes and the vacuum for the suction cups is activated so the fan can be picked up. The robot then moves vertically up by a fixed offset that gives enough space for the fan to unfold. By sending additional packets to the servo interface application on the raspberry the application makes the end-effector open and close the fan. The robot then puts the fan back onto the table by placing it slightly above the table surface and then disabling the vacuum suction cups and opening the gripper.

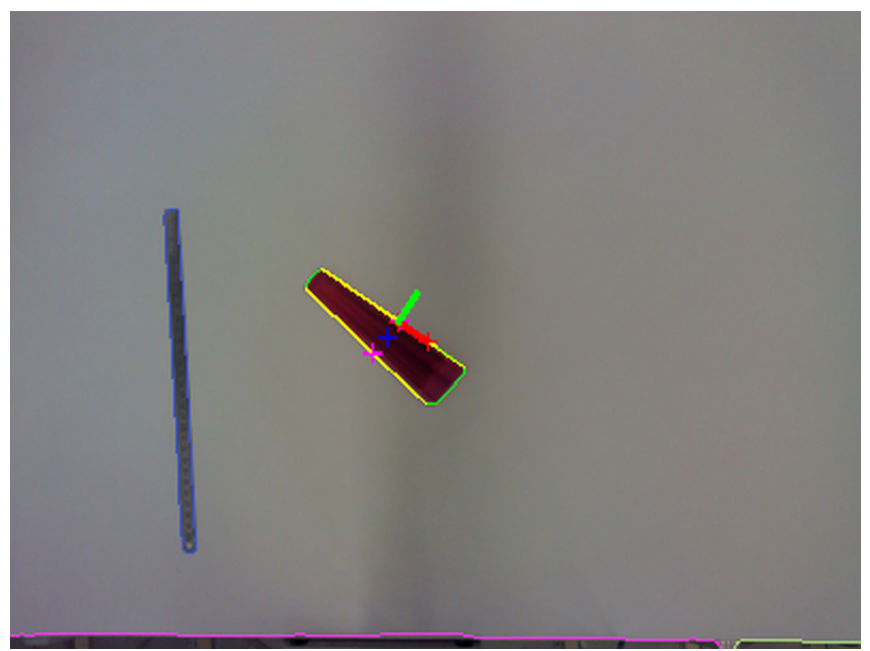

Fig. 9. Image with centroid (blue cross), hough lines (yellow) and proper coordinate system (red/green lines for $\mathrm{x}$ - and $\mathrm{y}$-axis). The pink crosses denote the base point of the plumb on the fan's boundary (hough lines).

In fig. 11, the process is displayed as an image sequence taken from the video we submitted. The robot is fitted to a welding table, which also serves as the surface for our experiment - we just added a wooden plate coated with white color. The camera is mounted on a cantilever built up by aluminum profiles, looking vertically down to the surface. Initially, the robot is outside of the camera's field of view so that it does not interfere with the image the camera captures. The fan is placed on the table in a random location and 


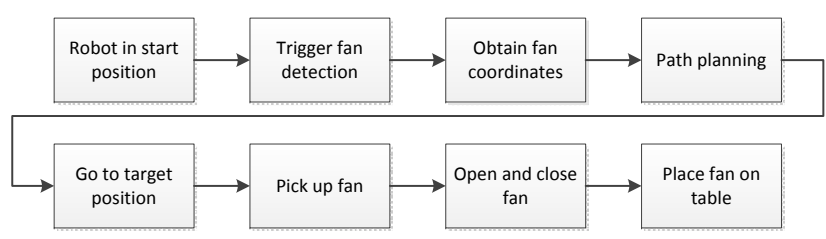

Fig. 10. Process Overview

orientation. However, this is limited by the workspace of the robot. Then the process is started by pushing a button on the robot control panel.

To evaluate the system performance, we conducted multiple test runs. We let the system do 5 series of runs where each series consisted of 10 complete runs. Between each run, we changed the location and orientation of the fan randomly but with respect to the workspace of the robot. We have found that the system works very reliable. Only in 2 out of the 50 runs the system was not able to detect the fan properly. The system failed to pick up the fan or place it back on the table properly in 3 cases. The overall time for one process turned out the be around 5 seconds in average. Yet we identified some potential for improvement that lies within the way the process is programmed: Picking up the fan, opening and closing it and putting it back was done purely consecutive. It should be possible to do this asynchronous, i.e. unfolding the fan as the robot moves up and closing it as the robot moves down. Moreover by using a stronger / faster servo the opening and closing of the fan could be speed up a little bit. We estimate this way we could possibly save up to 1.5 seconds.

\section{CONCLUSION}

The approach we have taken for the IROS 2018 fan challenge was driven by our field of research which is production automation. Our main design decision was to not mimic the human process but to built a custom endeffector based on the kinematics of the fan. To localize the fan we have implemented a camera-based detection system. We have found that the system works reliably and is able to complete the process in around 5 seconds. We suppose that we could reduce the time to around 3.5-4 seconds through further optimizations. The end-effector is able to repeatable open and close the fan. To sum up, we have shown that the system we have built is able to satisfy the objectives of the challenge: 1) pick up the closed fan from a table, 2) position the fan in parallel plane to the all, 3) open the fan, 4) close the fan, 5) place it on the table.

However, the design was only possible because of the very loose restrictions of the challenge. For instance, within our experiments the robot and the camera have been in a fixed location which limits the position of the fan to the field of view of the camera and the inherent workspace of the robot. To make it possible to pick up the fan in arbitrary locations from arbitrary surfaces, major modifications of the system would be necessary (e.g. use a mobile manipulator, mount the camera on the manipulator).

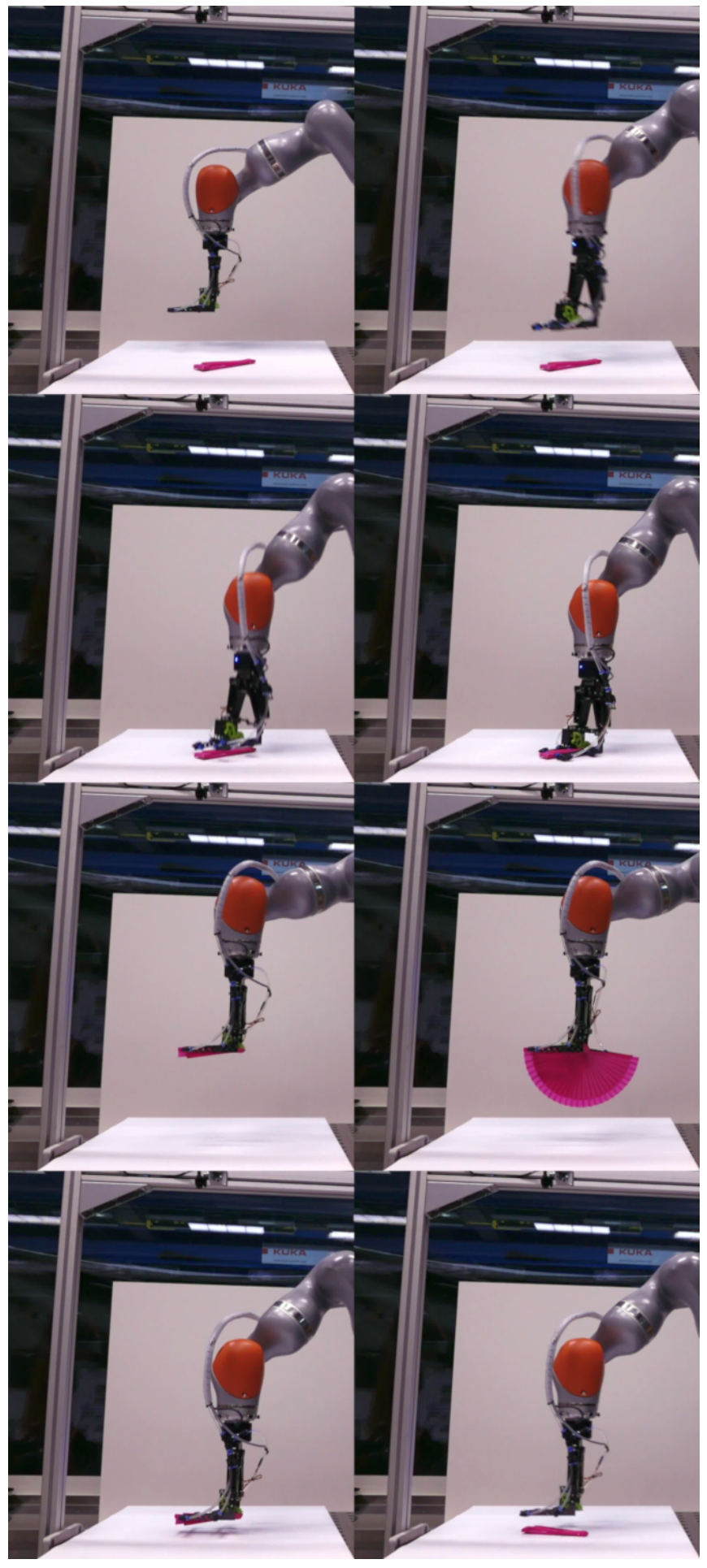

Fig. 11. Detection, gripping and fan operation 


\section{ACKNOWLEDGEMENT}

The authors acknowledge the financial support by the Federal Ministry for Economic Affairs and Energy (BMWi) of Germany.

\section{REFERENCES}

[1] D. H. Ballard, "Generalizing the hough transform to detect arbitrary shapes," in Readings in Computer Vision: Issues, Problems, Principles, and Paradigms, M. A. Fischler and O. Firschein, Eds. San Francisco, CA, USA: Morgan Kaufmann Publishers Inc., 1987, pp. 714-725. [Online]. Available: http://dl.acm.org/citation.cfm?id=33517.33574

[2] J. F. Canny, ",, a computational approach to edge detection”."

[3] Ethercat Technology Group, "Ethercat." [Online]. Available: https: //www.ethercat.org/default.htm

[4] A. Huamán, "„features2d + homography to find a known object”." [Online]. Available: \{\}http://docs.opencv.org/doc/tutorials/features2d/ feature_homography/feature_homography.html\#feature-homography

[5] iDS, "USB 2 uEye XS industrial camera." [Online]. Available: https://en.ids-imaging.com/store/products/cameras/ usb-2-0-cameras/ueye-xs.html

[6] F. Krebs, L. Larsen, G. Braun, and W. Dudenhausen, "Design of a multifunctional cell for aerospace CFRP production," in Lecture Notes in Mechanical Engineering, 2013.

[7] KUKA, "LBR iiwa 14 R820." [Online]. Available: https://www.kuka. com/en-us/products/robotics-systems/industrial-robots/lbr-iiwa

[8] — - "Sunrise OS." [Online]. Available: https://www.kuka.com/ en-us/products/robotics-systems/software/system-software/sunriseos

[9] opencv, "Homepage „OpenCV (Open Source Computer Vision)"," downloaded march 2018. [Online]. Available: http://www.opencv.org

[10] Robotiq, "Adaptive 2-Finger Gripper 140." [Online]. Available: https://robotiq.com/products/2-finger-adaptive-robot-gripper

[11] K. A. S. Suzuki, “,opological structural analysis of digital binary images by border following"." 